\title{
Autoantibodies against Glial Fibrillary Acidic Protein (GFAP) in Cerebrospinal Fluids from Pug Dogs with Necrotizing Meningoencephalitis
}

\author{
Masahiro SHIBUYA ${ }^{1)}$, Naoaki MATSUKI ${ }^{1) *}$, Kaori FUJIWARA ${ }^{1)}$, Shinobu IMAJOH-OHMI ${ }^{22}$, Hiroyuki FUKUDA ${ }^{2}$, \\ Ngoc T. PHAM ${ }^{1)}$, Satoshi TAMAHARA ${ }^{1)}$ and Kenichiro $\mathrm{ONO}^{1)}$ \\ ${ }^{1)}$ Department of Veterinary Clinical Pathobiology, Graduate School of Agricultural and Life Sciences, The University of Tokyo, 1-1-1 \\ Yayoi, Bunkyo-ku, Tokyo 113-8657 and ${ }^{2)}$ Laboratory Center for Proteomics Research, the Institute of Medical Science, The University of \\ Tokyo, 4-6-1 Shiroganedai, Minato-ku, Tokyo, 108-8639, Japan
}

(Received 10 August 2006/Accepted 17 November 2006)

\begin{abstract}
Cerebrospinal fluids (CSFs) from 9 Pug dogs with necrotizing meningoencephalitis (NME: Pug dog encephalitis) were examined to identify the antigens for anti-astrocyte autoantibodies. Each CSF exhibited a positive reaction to the cytoplasm of cultured canine astrocytes by an indirect fluorescent antibody test. In an immunoblotting analysis on normal canine brain proteins, eight of 9 CSFs showed a common band of $52 \mathrm{kDa}$, corresponding to glial fibrillary acidic protein (GFAP), and all of 9 CSFs reacted with purified bovine GFAP. From these results, GFAP is one of the common autoantigens in Pug dogs with NME. On the other hand, the reactivity of CSFs to chymotrypsin-digested bovine GFAP fragments were variable among dogs, indicating that the antibodies in the CSFs recognized different epitopes on GFAP.
\end{abstract}

KEY WORDS: autoantibody, canine, CSF, GFAP, NME.

J. Vet. Med. Sci. 69(3): 241-245, 2007

Canine necrotizing meningoencephalitis (NME: Pug dog encephalitis) is an idiopathic inflammatory disease in the brain, affecting specific small-sized breeds such as Pugs [4$6,18,22]$. Histopathology of NME is characterized by lymphocytic inflammation and subsequent necrosis in the cerebral cortex [20]. While the pathogenesis of NME is unclear, Uchida et al. [22] and our previous report [12] demonstrated that dogs with NME possessed anti-astrocyte autoantibodies in their cerebrospinal fluids (CSFs), suggesting that NME is an autoimmune disease. In addition, Suzuki et al. [20] reported the immunoglobulin and complement deposition on astrocytes in an NME case, supporting the pathological roles of anti-astrocyte autoantibodies. In the present study, we identified a common autoantigen for the anti-astrocyte autoantibodies in CSFs from Pug dogs with NME.

\section{MATERIALS AND METHODS}

CSFs: Between January 2001 to April 2004, 9 Pug dogs (11-month to 7-year of age, 5 males and 4 females) were referred to University of Tokyo Veterinary Medical Center, and were diagnosed as NME by magnetic resonance imaging (MRI) and postmortem pathological examinations. CSFs were collected from the cisterna magna under general anesthesia from 7 cases and during necropsy from 2 cases. A CSF sample from a clinically healthy Pug dog was used as a control.

Indirect fluorescent antibody test (IFA) on cultured astrocytes: Primary cultures of astrocytes were prepared from the forebrain of full-term fetuses of a clinically healthy Bea-

\footnotetext{
* CoRrespondence to: Matsuki, N., Department of Veterinary Clinical Pathobiology, Graduate School of Agricultural and Life Sciences, The University of Tokyo, 1-1-1 Yayoi, Bunkyo-ku, Tokyo 113-8657, Japan.
}

gle dog as described by MaCarthy et al. [14]. The purity of obtained astrocytes was confirmed by an immunofluorescence assay with an anti-human GFAP monoclonal antibody (YLEM, Roma, Italy). Over $99 \%$ of cultured cells were GFAP-positive astrocytes. Astrocytes were maintained in Dulbecco's Modified Eagle's Medium (SigmaAldrich, St. Louis, MO, U.S.A.) with $20 \%$ fetal calf serum (Invitrogen, Carlsbad, CA, U.S.A.) under 5\% $\mathrm{CO}_{2} / 95 \%$ air at $37^{\circ} \mathrm{C}$.

The reaction of CSFs on astrocytes was examined by an IFA test. That is, astrocytes were cultured on 22-mm-diameter glass coverslips for $48 \mathrm{hr}$, rinsed with HBSS, fixed with cold acetone for $5 \mathrm{~min}$ and dried. After 3 washes with phosphate-buffered saline (PBS), the cells were incubated with diluted CSF $(1: 10)$ at $37^{\circ} \mathrm{C}$ for $60 \mathrm{~min}$. After 3 washes, fluorescein isothiocyanate (FITC)-conjugated goat anti-canine IgG (Bethyl, Montogomery, TX, U.S.A.) was applied at $1: 400$ at $37^{\circ} \mathrm{C}$ for $60 \mathrm{~min}$. Then, the cells were washed, dried, mounted with Vectashield (Vector Labs, Burlingame, CA, U.S.A.), and analyzed by a fluorescent microscope (Optiphoto-2, Nikon, Tokyo, Japan) equipped with an image analyzing system (ORCA-1394, Hamamatsu Photonics, Hamamatsu, Japan).

Immunoblot on canine brain proteins: A piece of the cerebral cortex from a healthy female Beagle dog was kindly gifted from Professor Tsujimoto, Department of Veterinary Internal Medicine, The University of Tokyo. An approximate $1 \mathrm{~g}$ of the cerebral cortex was washed 3 times in ice-cold PBS and homogenized in $10 \mathrm{~m} l$ of sucrose buffer (250 mM sucrose, $1 \mathrm{mM}$ EDTA, $1.6 \mathrm{mM}$ phenylmethanesulfonyl fluoride, $10 \mu \mathrm{g} / \mathrm{m} l$ pepstatin A, $10 \mu \mathrm{g} / \mathrm{m} l$ leupeptin, $10 \mu \mathrm{g} / \mathrm{m} l$ aprotinin, $\mathrm{pH}$ 7.4) on ice. The protein concentration of the homogenate was determined with a commercial kit (Protein Assay kit, Bio-Rad Laboratories, Hercules, CA, 
U.S.A.), using bovine serum albumin as a standard.

For electrophoresis, approximately $10 \mu \mathrm{g}$ of brain proteins were separated by sodium dodecyl sulfate-polyacrylamide gel electrophoresis (SDS-PAGE) on 10\% gels, and then stained with Coomassie Brilliant Blue or transferred onto nitrocellulose membranes (Hybond ECL, Amersham Pharmacia Biotech, Piscataway, NJ, U.S.A.). The membranes were blocked by 5\% skim milk (Becton Dickinson, Sparks, MD, U.S.A.) in Tris-buffered saline containing $0.1 \%$ Tween 20 (TBST) for $1 \mathrm{hr}$, and incubated with diluted CSFs (1:200) for $1 \mathrm{hr}$. After washes, autoantibody was reacted with horseradish peroxidase (HRP)-conjugated goat anti-canine $\operatorname{IgG}(1: 4,000$, Bethyl) for $1 \mathrm{hr}$ and visualized by a chemiluminescence kit (ECL and Hyperfilm ECL, Amersham).

Mass spectrometry of proteins: Protein bands on stained gels, which corresponded to positive bands on the immunoblot membranes, were recovered, in-gel digested with trypsin, and identified with matrix-assisted laser desorption/ ionization-time of flight (MALDI-TOF) tandem mass spectrometry (MS/MS: Model 4700 Proteomics Analyzer, Applied Biosystems, U.S.A.) as previously described [15]. The MS/MS spectra were analyzed by using the Mascot software program (Matrix Science, London, UK).

Immunoblot on intact/digested bovine GFAP: One $\mu \mathrm{g} /$ lane of intact bovine GFAP (Progen Biotechnik, Heidelberg, Germany) was separated by SDS-PAGE on $10 \%$ gels and was transferred onto nitrocellulose membranes. Digestion of bovine GFAP by $\alpha$-chymotrypsin (Sigma-Aldrich: GFAP to enzyme weight ratio was 5:1) was performed in 10 $\mathrm{mM}$ Tris ( $\mathrm{pH} 8.8$ ) on ice for $30 \mathrm{~min}$. The digests were separated by SDS-PAGE on $13.5 \%$ gels, and were transferred onto nitrocellulose membranes. These membranes were submitted to immunostaining with CSFs as described above.

\section{RESULTS}

Distribution of autoantigen(s) in cultured astrocytes: Figure 1 shows the results of IFA tests on cultured canine astrocytes. All CSFs from NME cases clearly reacted with astrocytes, showing the positive signal in the cytoplasm within the cell body and processes. The reaction pattern of CSFs was similar to that of an anti-human GFAP monoclonal antibody (mAb), whereas the CSF from the healthy control(C) did not react with astrocytes.

Immunoblot on canine brain proteins and mass spectrometry of proteins: Results of immunoblotting on canine brain proteins are shown in Fig. 2. In case 1-7 and 9, a common band of $52 \mathrm{kDa}$ was detected (arrow), which corresponded to GFAP by the amino acid sequence analysis with TOFMASS (Table 1). In some cases, additional bands were observed at different molecular weights (arrow heads).

Immunoblot on intact/digested bovine GFAP: Figure 3 shows the results of immunoblotting on intact bovine GFAP. All CSFs from NME cases (1-9), as well as antihuman GFAP monoclonal antibody (mAb), clearly reacted to bovine GFAP at approximately $50 \mathrm{kDa}$, whereas neither

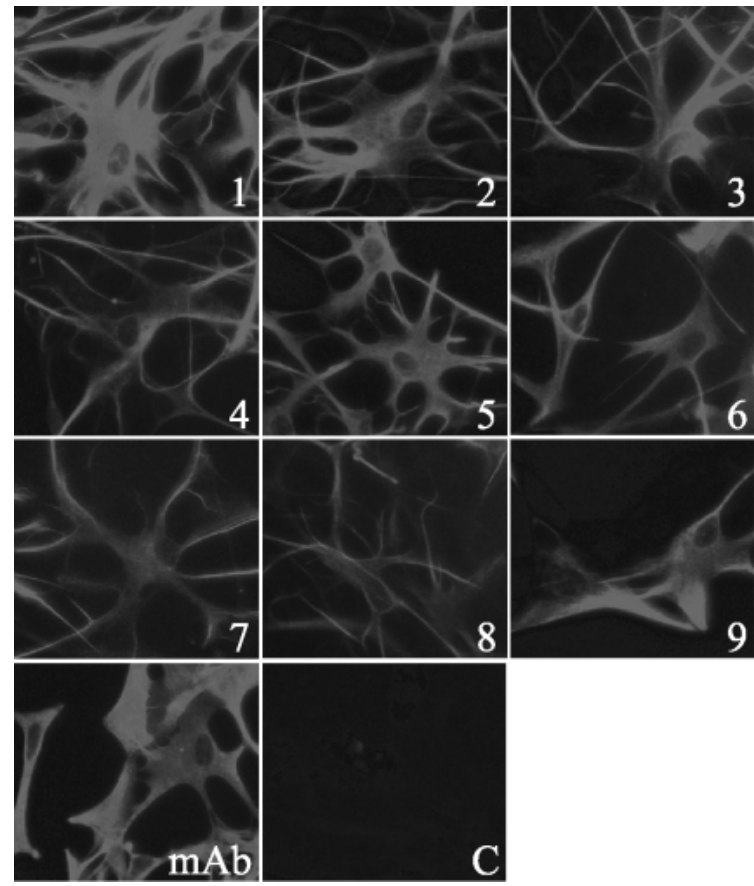

Fig. 1. Indirect fluorescent antibody test (IFA) on cultured canine astrocytes. All CSFs from NME cases (1-9) clearly reacted to astrocytes, showing positive signals in the cytoplasm within the cell body and processes. The reaction patterns were similar to that of anti-human GFAP monoclonal antibody $(\mathrm{mAb})$, whereas no reaction was observed in CSF from a healthy control (C).

the control CSF (C) nor second antibody alone (N: goat anticanine $\operatorname{IgG}$ ) reacted to bovine GFAP.

CSFs from 4 cases (case 4-7) were examined on digested GFAP to evaluate the epitopes on GFAP (Fig. 4). In all of 4 cases, a common band was observed at $25 \mathrm{kDa}$. On the other hand, CSF of case 6 strongly reacted to the digested GFAP fragment at approximately $16 \mathrm{kDa}$ (arrow head). The band patterns were variable among tested dogs.

\section{DISCUSSION}

The present study was designed to identify the autoantigen for anti-astrocyte autoantibodies, which were frequently detected in the CSF from NME cases [12]. All of the present 9 cases also possessed anti-astrocyte autoantibodies, which were detected by an IFA test on frozen sections of the normal canine cerebrum (data not shown). The IFA test on cultured fetal canine astrocyte was done to evaluate the distribution of autoantigen(s) in astrocytes. All CSFs strongly reacted to the cytoplasm of astrocytes, while minimal reaction was detected on the plasma membrane. To identify the cytoplasmic autoantigen, an immunoblot on normal canine brain proteins was performed, and GFAP was one of the major candidates of autoantigens. The reactivity of CSFs with GFAP was well confirmed by the immunoblotting on purified bovine GFAP. On the other hand, the reactivity of 


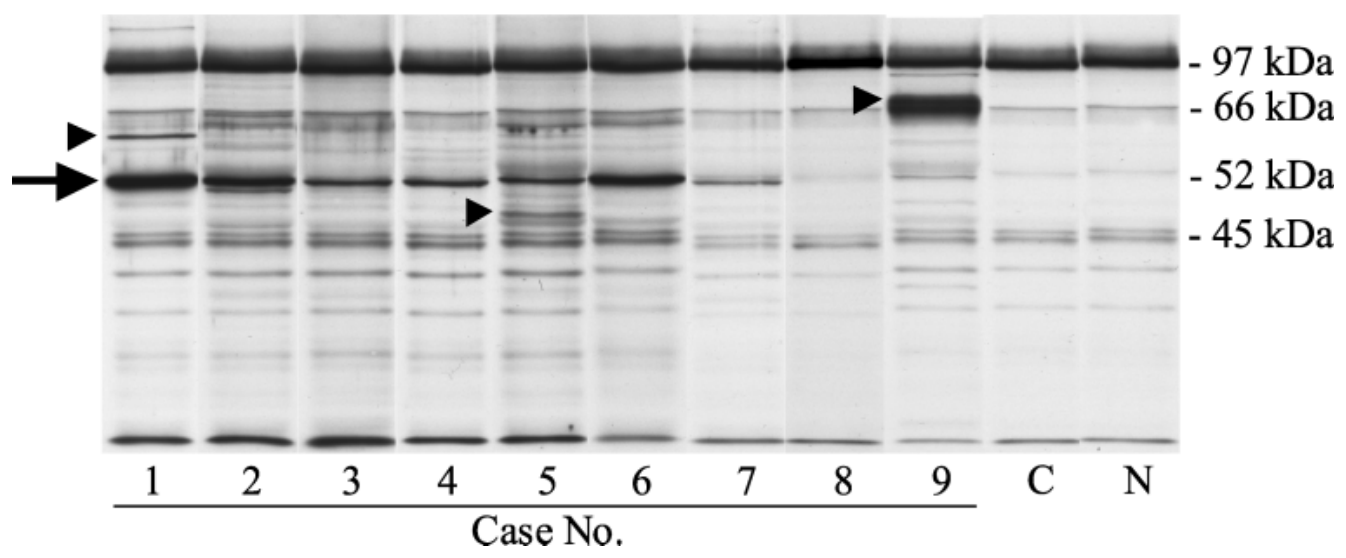

Fig. 2. Immunoblot on canine brain proteins with CSFs from NME cases (1-9), the control CSF (C) and the second antibody alone (N). In case 1-7 and 9, a common band at approximately $52 \mathrm{kDa}$ was detected (arrow), which corresponded to GFAP. In some cases, additional bands were observed at different molecular weights (arrow heads).

Table 1. Tryptic peptides of a common band reacted with CSFs and identified as GFAP by mass spectrometry

\begin{tabular}{cccccc}
\hline \multirow{2}{*}{ No. } & \multicolumn{2}{c}{ Mass } & \multicolumn{2}{c}{ Position (residue) } & \multirow{2}{*}{ Matched peptides } \\
\cline { 2 - 5 } & Observed & Calculated & Start & End & \\
\hline 1 & 1098.63 & 1097.62 & 96 & 105 & ALAAELNQLR \\
2 & 1177.63 & 1176.61 & 112 & 121 & LADVYQAELR \\
3 & 1265.65 & 1264.64 & 259 & 270 & SKFADLTDAAAR \\
4 & 1050.56 & 1049.51 & 261 & 270 & FADLTDAAAR \\
5 & 1060.53 & 1059.48 & 280 & 287 & HEANDYRR \\
6 & 1208.58 & 1207.58 & 320 & 330 & EAASYQEALAR \\
7 & 1499.8 & 1498.78 & 345 & 356 & HLQEYQDLLNVK \\
\hline
\end{tabular}

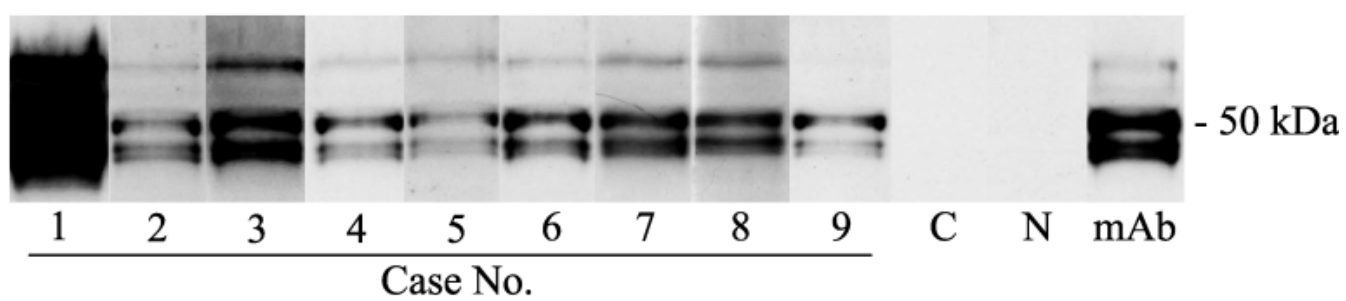

Fig. 3. Immunoblot on intact bovine GFAP. All CSFs from NME cases (1-9), as well as anti-human GFAP monoclonal antibody $(\mathrm{mAb})$, clearly reacted to bovine GFAP at $50 \mathrm{kDa}$, while neither the control CSF (C) nor the second antibody alone $(\mathrm{N})$ reacted to bovine GFAP.

CSFs to bovine GFAP digested by $\alpha$-chymotrypsin were variable among dogs, suggesting the antibodies in those CSFs recognized different epitopes on GFAP. In humans, only one patient with systemic lupus erythematosus (SLE) has been reported to be positive for an anti-GFAP autoantibody in the CSF [19], although the anti-GFAP autoantibodies are often detected in human sera. In the present study, we detected anti-GFAP autoantibodies in the CSFs from all NME cases, indicating a unique feature of canine NME.

GFAP is the major protein of intermediate filaments in mature astrocytes [7], and is widely used as a marker of astrocytes [9]. Studies on GFAP reported that GFAP has significant roles in maintaining the morphology of astrocytes [8], forming the integrity of blood-brain barrier [11], and modulating the synaptic efficacy [13]. Pekny and Pekna [17] reported that GFAP has essential roles in reactive gliosis during central nervous system (CNS) pathologies, and in nerve regeneration after brain trauma. In addition, human GFAP gene mutations have been reported in fatal [2] and infant [1] CNS diseases.

The present study does not provide information whether the anti-GFAP autoantibody is a cause, consequence or 


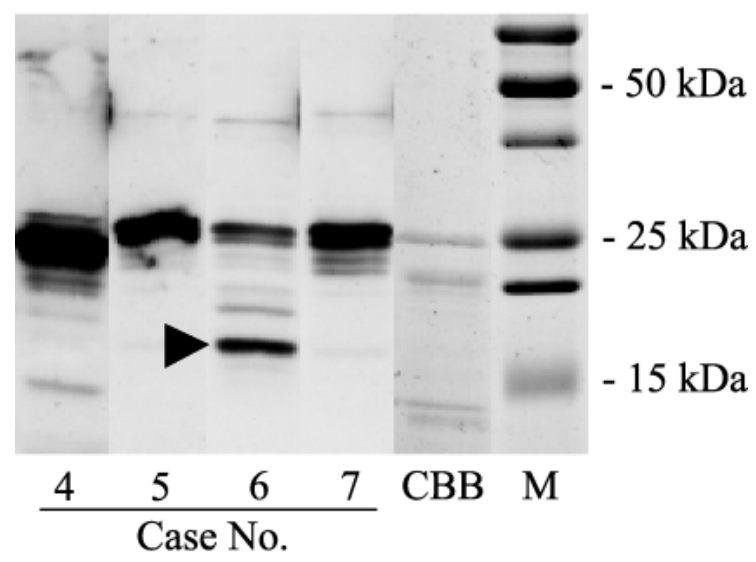

Fig. 4. Immunoblot on digested bovine GFAP. CSFs from 4 cases (case 4-7) were examined. Although a common band was observed at $25 \mathrm{kDa}$, the band patterns were variable. Case 6 strongly reacted to the fragment of $16 \mathrm{kDa}$ (arrow head). CBB; digested GFAP stained by Coomassie Brilliant Blue, $\mathrm{M}$; molecular weight markers.

epiphenomenon of canine NME. Production of anti-GFAP autoantibody may be caused by GFAP leakage from damaged astrocytes, since GFAP is the principal cytoplasmic protein in mature astrocytes. However, autoantibodies against cytoplasmic autoantigens were also detected in autoimmune hepatitis [23] and type I diabetes [21] in human patients, and were considered to play essential roles in the pathogenesis of diseases. In canine NME, IgG and complement deposition was detected on astrocytes, suggesting a possibility of autoantibodies to disrupt the astrocyte functions [20]. Astrocyte dysfunctiton was reported to induce disturbances in the CNS, since astrocytes modulate the extracellular concentrations of excitatory amino acids such as glutamate $[10,16]$. Excessive accumulation of glutamate in the extracellular space induces neuronal cell death by the excitatory neurotoxicity [3].

In conclusion, GFAP is one of common autoantigens in canine NME, and the anti-GFAP autoantibodies in the CSF may be closely related to the pathogenesis and/or pathophysiological states of NME, although further studies are necessary to clarify the underlying pathological mechanisms.

\section{REFERENCES}

1. Alexander, W. 1949. Progressive fibrinoid degeneration of fibrillary astrocytes associated with mental retardation in a hydrocephalic infant. Brain 72: 373-381.

2. Brenner, M., Johson, B. A., Boespflug-Tanguy, O., Rodriguez, D., Goldman, E. J. and Messing, A. 2001. Mutations in GFAP, encoding glial fibrillary acidic protein, are associated with Alexander disease. Nature Genet. 27: 117-120.

3. Choi, D. 1998. Glutamate neurotoxicity and diseases of the nervous system. Neuron 1: 623-634.

4. Cordy, D. R. and Holliday, T. A. 1989. A necrotizing meningoencephalitis of Pug dogs. Vet. Pathol. 26: 191-194.
5. de Lahunta, A. 1983. Chronic encephalitis of Pug dogs. pp. 384-385. In: Veterinary Neuroanatomy and Clinical Neurology, 2 ed. (de Lahunta, A. ed.), WB Saunders, Philadelphia, PA, U.S.A.

6. Ducote, M. J., Johnson, E. K., Dewey, W. C., Walker, A. M., Coates, R. J. and Berridge, R. B. 1999. Computed tomography of necrotizing meningoencephalitis in 3 Yorkshire Terriers. Vet. Radiol. Ultrasound 40: 617-621.

7. Eng, F. L. 1985. Glial fibrillary acidic protein (GFAP): the major protein of glial intermediate filaments in differentiated astrocytes. J. Neuroimmunol. 8: 203-214.

8. Ghirnikar, R., Yu, A. and Eng, L. 1994. Astrogliosis in culture: III. Effect of recombinant retrovirus expressing antisense glial fibrillary acidic protein RNA. J. Neurosci. Res. 38: 376-385.

9. Goebel, H., Schlie, M. and Eng, L. 1987. Immunohistopathologic spectrum of the glial fibrillary acidic protein in neurooncology. Acta Histochem. (Suppl.) 34: 81-93.

10. Henn, F. and Hamberger, A. 1971. Glial cell function: uptake of transmitter substances. Proc. Natl. Acad. Sci. U. S. A. 68: 2686-2690.

11. Liedtke, W., Edelmann, W., Bieri, L. P., Chiu, F-C., Cowman, J. N., Kucherlapati, R. and Raine, S. C. 1996. GFAP is necessary for the integrity of CNS white matter architecture and long-term maintenance of myelination. Neuron 17: 607-615.

12. Matsuki, N., Fujiwara, K., Tamahara, S., Uchida, K., Matsunaga, S., Nakayama, H., Doi, K., Ogawa, H. and Ono, K. 2004. Prevalence of autoantibody in cerebrospinal fluids from dogs with various CNS diseases. J. Vet. Med. Sci. 66: 295-297.

13. McCall, A. M., Gregg, G. R., Behringer, R. R., Brenner, M., Delaney, G. C., Galbreath, J. E., Zhang, L. C., Pearce, A. R., Chiu, Y. S. and Messing, A. 1996. Targeted deletion in astrocyte intermediate filament (Gfap) alters neuronal physiology. Proc. Natl. Acad. Sci. U. S. A. 93: 6361-6366.

14. McCarthy, K.D. and de Vellis, J. 1980. Preparation of separate astroglial and oligodendroglial cell cultures from rat cerebral tissue. J. Cell Biol. 85: 890-902.

15. Ogino, T., Fukuda, H., Imajoh-Ohmi, S., Kohara, M. and Nomoto, A. 2004. Membrane binding properties and terminal residues of the mature Hepatitis C Virus capsid protein in insect cells. J. Virol. 78: 11766-11777.

16. Pekny, M., Eliasson, C., Siushansian, R., Ding, M., Dixon, J. S., Pekna, M., Wilson, X. J. and Hamberger, A. 1999. The impact of genetic removal of GFAP and/or vimentin on glutamine levels and transport of glucose and ascorbate in astrocytes. Neurochem. Res. 24: 1357-1362.

17. Pekny, M. and Pekna, M. 2004. Astrocyte intermediate filaments in CNS pathologies and regeneration. J. Pathol. 204: 428-437.

18. Stalis, I. H., Chadwick, B., Dayrell-Hart, B., Summers, B. A. and Van Winkle, T. J. 1995. Necrotizing meningoencephalitis of Maltese dogs. Vet. Pathol. 32: 230-235.

19. Stuart, B. M. and Gregson, N. A. 1998. Cerebral calcification in a patient with systemic lupus erythematosus and a monoclonal IgG reactive with glial fibrillary acidic protein. $B r . J$. Rheumatol. 37:1355-1357

20. Suzuki, M., Uchida, K., Morozumi, M., Hasegawa. T., Yanai, T., Nakayama, H. and Tateyama, S. 2003. A comparative pathological study on canine necrotizing meningoencephalitis and granulomatous meningoencephalomyelitis. J. Vet. Med. Sci. 65: 1233-1239.

21. Takino, H., Kawasaki, E., Yano, M., Matsumoto, K., Uotani, S., Okuno, S., Takao, Y., Yamasaki, H., Yamaguchi, Y., Akazawa, S. and Nagataki, S. 1994. The heterogeneity of whole 
islet cell cytoplasmic antibodies evidenced by absorption test with glutamic acid decarboxylase. J. Autoimmun. 7: 389-398.

22. Uchida, K., Hasegawa, T., Ikeda, M., Yamaguchi, R. and Tateyama, S. 1999. Detection of autoantibody from Pug dogs with necrotizing encephalitis (Pug dog encephalitis). Vet.

\section{Pathol. 36: 301-307.}

23. Zachou, K., Rigopoulou, E. and Dalekos, G. 2004. Autoantibodies and autoantigens in autoimmune hepatitis: important tools in clinical practice and to study pathogenesis of the disease. J. Autoimmune Dis. 1: 2. 